\title{
Layered Surface Detection for Virtual Unrolling
}

Dahl, Vedrana Andersen; Dahl, Anders Bjorholm; Trinderup, Camilla Himmelstrup; Gundlach, Carsten

\section{Published in:}

Proceedings of 24th International Conference on Pattern Recognition

Link to article, DOI:

10.1109/ICPR.2018.8545715

Publication date:

2018

Document Version

Peer reviewed version

Link back to DTU Orbit

Citation (APA):

Dahl, V. A., Dahl, A. B., Trinderup, C. H., \& Gundlach, C. (2018). Layered Surface Detection for Virtual Unrolling. In Proceedings of 24th International Conference on Pattern Recognition (pp. 3074-3080). IEEE. International Conference on Pattern Recognition https://doi.org/10.1109/ICPR.2018.8545715

\section{General rights}

Copyright and moral rights for the publications made accessible in the public portal are retained by the authors and/or other copyright owners and it is a condition of accessing publications that users recognise and abide by the legal requirements associated with these rights.

- Users may download and print one copy of any publication from the public portal for the purpose of private study or research.

- You may not further distribute the material or use it for any profit-making activity or commercial gain

- You may freely distribute the URL identifying the publication in the public portal 


\section{Layered Surface Detection for Virtual Unrolling}

\author{
Vedrana Andersen Dahl, \\ Anders Bjorholm Dahl \\ and Camilla Himmelstrup Trinderup \\ Department of Applied Mathematics and Computer Science \\ Technical University of Denmark \\ Lyngby, Denmark \\ Email: \{vand,abda,ctri\}@dtu.dk
}

\author{
Carsten Gundlach \\ Department of Physics \\ Technical University of Denmark \\ Lyngby, Denmark \\ Email: cagu@fysik.dtu.dk
}

\begin{abstract}
We present a method for virtual unrolling of a thin rolled object. From a volumetric image of the rolled object we obtain a flat image of the object's surface, which allows visual inspection of the object and has a number of applications. Our method exploits the geometric constrains of the problem and detects a single rolled surface. For surface detection we adapt a solution to an optimal net surface problem, previously used for terrain-like and tubular surfaces. We present our approach on an example of a rolled sheet of microelectronic, which has a layer of flexible polymer substrate and a thin metal layer lithographically coated onto the polymer. Our approach is automatic and robust. The unrolled image is undistorted, and the surface structures may be accurately quantified making our approach a good candidate for an industrial application of virtual unrolling.
\end{abstract}

\section{INTRODUCTION}

Virtual unrolling is the process of transforming a volumetric image of a rolled object to an image of the unrolled object. This has a number of applications, and has gained significant interest for viewing scrolled historical parchments, which are too fragile to be physically unwrapped. Carbonized scrolls of ancient papyri are particularly fragile and here virtual unrolling from X-ray micro computed tomography (CT) [1] reveals inscriptions that can otherwise not be seen [2], [3], [4].

Imaging rolled objects and unrolling them virtually can also be used for industrial applications, and in this paper we apply virtual unrolling to a sheet of microelectronics, which has a thin conductive metal layer that is lithographically coated onto a flexible polymer substrate. The sheet is rolled, which might introduce cracks in the conductive layer and influence the properties of the device. Virtual unrolling is used to visually inspect and quantify the cracks. Another example is cylindrical rechargeable batteries, where the common design involves a rolled layered structure. Battery properties depend on the surface conditions of the anode and cathode layers and these can be characterised through virtual unrolling.

Virtual unrolling brings possibilities in volumetric imaging, also for applications outside cultural heritage or industrial inspection. When imaging a flat (not rolled) object using volumetric techniques, like micro-CT as we use here, a large part of the imaged volume is just air, and the object occupies a very limited part of the space. However, by physically rolling the object and adjusting the field of view, the object may take up more of the imaged space. As such, we obtain a higher physical resolution with smaller voxels and/or a reduced scanning time.

The research community working with virtual unrolling for cultural heritage applications has paid most attention to the choice of imaging techniques and modality [5], [6], and only limited focus is given to automated and reliable image processing [7]. Therefore, most proposed methods require intense manual input, which is justified when processing few and unique data sets. However, if applying virtual unrolling to applications like the one presented here, robustness and automation is imperative. Only automated and robust methods will allow assessing a statistical representative set of samples, and obtaining measurements (related to the material properties) with a high confidence.

Some earlier work on virtual unrolling like [8] attempted a difficult problem of a densely packed ancient scroll imaged with micro-CT. Their conclusion was that virtual unrolling is only possible using manual annotation. Because of the required effort in manual input, the obtained virtual unrolling has limited use. A semi-automated approach was proposed in [9] based on a manual initialization, but also with a much easier problem compared to [8].

The basic idea for automating the unrolling in [9] is to segment the rolled object from the background and obtain the unrolled surface by skeletonization. A similar approach is used in [7] that extends the work in [10]. The main problem with this approach is, that the segmentation does not distinguish touching surfaces, which is a common case for rolled objects. In [7] they solve the problem of touching surfaces by improving the segmentation and employing a heuristic for separating the densely packed layers.

It should be noted, that both in the segmentation step, and in the following separation step, the algorithm makes binary decisions, and an introduced mistake is propagated through the pipeline. With densely rolled objects without air between the layers, a segmentation-based approach would also fail, because there would be no voids to separate the layers.

To overcome the potential problems in employing a segmentation followed by a post processing steps, we have chosen to employ a geometric model of the rolled object. Our model expects a layered structure and relies on an intensity change on the boundary between subsequent layers, which is typically 
present in micro-CT scans of rolled objects.

In our approach, we adapt the optimal net surface detection via graph search originally suggested by $\mathrm{Wu}$ and Chen [11]. They construct a graph on a set of sample points from a volume, such that the roughness of possible solutions is constrained. The optimality of the solution is defined in terms of a volumetric cost function derived from the data. The approach has been extended for finding multiple interrelated layered terrain-like and tubular surfaces [12], which made it applicable for medical image segmentation [13], [14] and led to further extensions. The volumetric cost function, which determines an optimal placement of the surface, originally defined only in terms of on-surface appearance, has been extended to incorporate appearance of the regions between surfaces [15], [16]. Furthermore, surface roughness has been allowed to vary across the surface [17], and soft shape constraints have been introduced [18].

\section{METHOD}

Our approach to virtual unrolling is geometrical, and we exploit the fact that the surface we are detecting is rolled. This constrains the solution space of the optimization problem and gives the robustness to our method.

We will first review an algorithm for finding optimal layered surfaces, with the focus on the inputs and the outputs. For details on how this algorithm works, the reader is referred to [11], [12], [19]. We also briefly cover the principle of transforming the data into volumetric cost, which is the input to the layered surface detection algorithm. Next we bring the core of our method: an explanation of how optimal layered surface detection may be used for virtual unrolling in general. Lastly, we bring some implementational choices relevant for unrolling microelectronic components.

\section{A. Layered surface detection, a review of the algorithm}

In a discrete volume $x \in\{1, \ldots, X\}, y \in\{1, \ldots, Y\}, z \in$ $\{1, \ldots, Z\}$, a terrain-like surface $s$ defined by $z=s(x, y)$ meets the smoothness constraint $\left(\Delta_{x}, \Delta_{y}\right)$ if

$|s(x, y)-s(x-1, y)| \leq \Delta_{x} \quad$ and $\quad|s(x, y)-s(x, y-1)| \leq \Delta_{y}$.

For a cost volume $c(x, y, z)$, an on-surface cost of $s$ is defined as

$$
C_{\text {on }}(s, c)=\sum_{x=1}^{X} \sum_{y=1}^{Y} c(x, y, s(x, y)) .
$$

The optimal net surface problem is concerned with finding a terrain-like surface with a minimum cost among all surfaces satisfying the smoothness constraint.

The polynomial time solution presented in [11] transforms the optimal net surface problem into that of finding a minimum-cost closed set [20] in a node-weighted directed graph with nodes representing volume voxels. This is further transformed into a problem of finding a minimum-cost $s$ - $t$ cut [20], [21] in a related arc-weighted directed graph. Minimumcost $s-t$ cut can be solved in polynomial time and efficiently found using algorithm of Boykov and Kolmogorov [22], a well known tool for many image segmentation tasks. While the optimal net surface problem is ultimately solved using the minimum-cost $s$ - $t$ cut algorithm, it should be noted that the graph constructed for surface detection is rather different than in examples from [22].

The extension to multiple surfaces developed in [12] may be exemplified by considering two terrain-like surfaces $s_{1}$ and $s_{2}$. The surfaces are said to meet an overlap constraint $\left(\delta_{\text {low }}, \delta_{\text {high }}\right)$ if

$$
\delta_{\text {low }} \leq\left|s_{2}(x, y)-s_{1}(x, y)\right| \leq \delta_{\text {high }} .
$$

Given two cost volumes $c_{1}$ and $c_{2}$ the total cost associated with surfaces $s_{1}$ and $s_{2}$ is

$$
C_{\text {on }}\left(s_{1}, c_{1}\right)+C_{\text {on }}\left(s_{2}, c_{2}\right)
$$

and the optimal surface detection will return a pair of surfaces with a minimum cost among all surfaces satisfying the overlap and smoothness constraints. Depending on the problem at hand, $c_{1}$ and $c_{2}$ may be different or identical, and likewise smoothness constrains may vary or be the same for the two surfaces.

Finally, two layered (i.e. non-intersecting and ordered) surfaces give rise to an in-region cost corresponding to the volume between two surfaces, and defined by

$$
C_{\text {in }}\left(s_{1}, s_{2}, c_{1,2}\right)=\sum_{x=1}^{X} \sum_{y=1}^{Y} \sum_{z=s_{1}(x, y)+1}^{s_{2}(x, y)} c_{1,2}(x, y, z) .
$$

This cost, together with the cost for the region under the surface $s_{1}$ and the region over the surface $s_{2}$, can be incorporated into the minimization problem [15]. We use the notation $c_{0,1}$ and $c_{2,3}$ for the cost volumes in connection with the boundary regions.

To summarize, for finding $K$ cost-optimal layered surfaces we need to define

- $K$ on-surface cost volumes $c_{k}, k=1, \ldots, K$, and/or

- $K+1$ in-region cost volumes $c_{k, k+1}, k=0, \ldots, K$.

The set of feasible surfaces is given by

- $K$ smoothness constraints $\left(\Delta_{x}^{k}, \Delta_{y}^{k}\right), k=1, \ldots, K$, and

- $K-1$ overlap constraints $\left(\delta_{\text {low }}^{k, k+1}, \delta_{\text {hight }}^{k, k+1}\right), k=$ $1, \ldots, K-1$.

Layered surface detection has found an immediate use for detecting tubular surfaces. The main principle is the fact that a circle $x^{2}+y^{2}=\rho^{2}$ appears as a straight line $r=\rho$ when represented in polar $(r, \theta)$ coordinates, see the top row of Fig. 1. For that reason, detecting a tubular surface is achieved by representing the volumetric data in a cylindrical coordinate system $(r, \theta, z)$ with the longitudinal axis $r=0$ roughly aligned with the center of the tube. We call this transformation unwrapping the volume, and to stress the discrete nature of surface detection we also say that the volume is sampled along the radial rays. Here the radial and the angular resolution are important practical parameters for unwrapping. In the unwrapped representation, the tubular surface is terrain-like and can be defined as $r=s(\theta, z)$. When using layered surface 
detection for tubular surfaces, additional constraints are added to ensure a smooth transition over $\theta=0$.

\section{B. Constructing cost volumes for surface detection}

The surfaces returned by the layered surface detection described in Sec.II-A are optimal in terms of the volumetric cost. Therefore, to use the surface detection algorithm we need to define a cost volume $c_{k}$ which takes small values where the data $V(x, y, z)$ supports the surface $k$. This modelling step, crucial for the performance of the algorithm, depends on the appearance of the surface in the volumetric data. The strategy in general involves transforming the volumetric data such that voxels of the surface attain the small values and here we describe the most common approaches.

If the surface to be detected is characterized by a certain voxel intensity $v_{s}$, then the cost volume may be simply defined as $\left(V-v_{s}\right)^{2}$. A related approach, inspired by the normal probability distribution, uses $1-\exp \left(-\left(V-v_{s}\right)^{2}\right)$.

A different strategy involves defining the cost volume in terms of a change in intensity. This is often advantageous, for example when we are interested in detecting a terrain-like surface on a boundary between a brighter region below and a darker region above. For this problem, a suitable cost volume is the gradient in the $z$ direction $\partial V / \partial z$, which attains small (negative) values at the surface. When computing intensity changes for tubular surfaces, the best approach is to first unwrap the volume, and then compute the gradient in the $r$ direction. For stability, a certain degree of Gaussian filtering might be applied to the unwrapped image. Equivalently, filtering the image with the difference of Gaussian yields a cost function robust to noise. This strategy is used for our unrolling of microelectronics.

\section{Virtual unrolling, the general principle}

A detection of the rolled surface bears resemblance to the detection of the layered tubular surfaces. In a similar way, the method involves unwrapping the volume, i.e. representing a $(x, y, z)$ volumetric data in a $(r, \theta, z)$ cylindrical coordinate system with the $r=0$ axis aligned with the center of the roll. The explanation of the general principle is based on a plane orthogonal to the longitudinal axis, denoted a slice through the volume.

While closed tubular surface appears predominately flat in a cylindrical coordinate system, rolled surface appears as a collection of slanted layers, depending on the number of times the surface is rolled, as illustrated in the bottom row of Fig. 1. Furthermore, rolled surface starts at a certain angle $\theta_{\mathrm{A}}$ and ends at angle $\theta_{\mathrm{B}}$. To account for this, we divide a full circle $[0,2 \pi\rangle$ into two parts, denoted $\left[\theta_{\mathrm{A}}, \theta_{\mathrm{B}}\right\rangle$ and $\left[\theta_{\mathrm{B}}, \theta_{\mathrm{A}}\right\rangle$, where one of those angular intervals wraps over $\theta=0$. Depending on the number of times the surface is rolled, we have $K+1$ layers in the first interval, and $K$ layers in the second interval. The illustration in Fig. 1 shows a situation with $K=1$, as there are two layers in $\left[\theta_{\mathrm{A}}, \theta_{\mathrm{B}}\right\rangle$ (which wraps over $\theta=0$ ), and one layer in $\left[\theta_{\mathrm{B}}, \theta_{\mathrm{A}}\right\rangle$. As such, we transform the problem
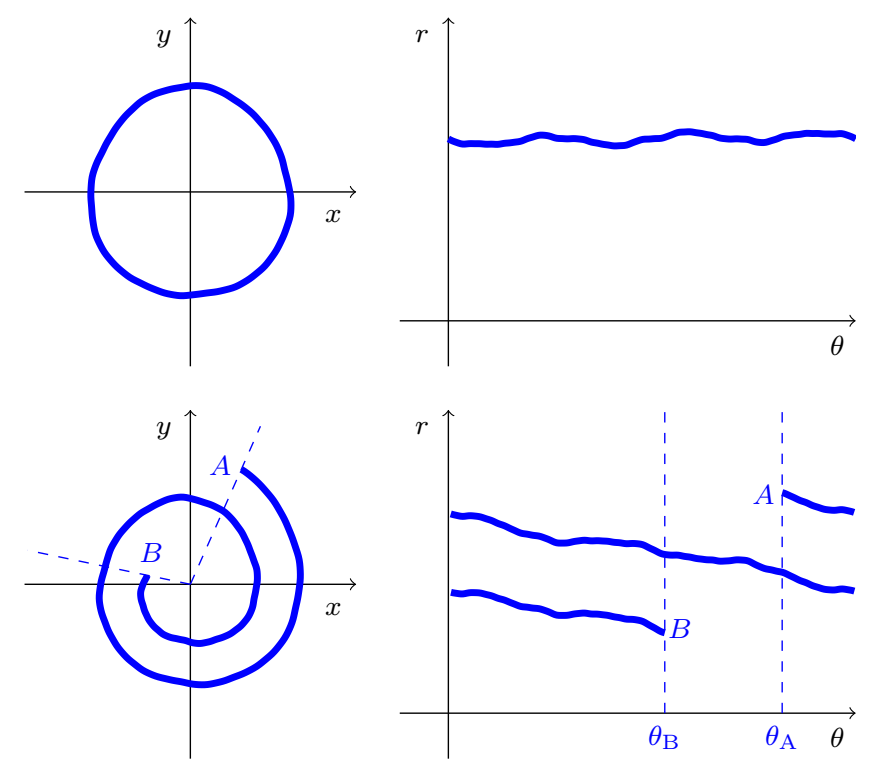

Fig. 1. Unwrapping a tubular (top row) and a rolled (bottom row) surface, illustrated on one slice. Note that $\theta$ is increasing in the clockwise direction (not to introduce a flip).

of detecting a rolled surface, into a problem of (two times) detecting layered surfaces.

This split problem is solved using the layered surface detection: the angular intervals $\left[\theta_{\mathrm{A}}, \theta_{\mathrm{B}}\right\rangle$ and $\left[\theta_{\mathrm{B}}, \theta_{\mathrm{A}}\right\rangle$ are discretized (the number of points in two intervals should be proportional to the length of the interval), and for every angle $\theta$ we use layered surface detection to identify $K+1$ or $K$ radii $r$. Finally, we re-connect the detected layers in an appropriate way to obtain a single (un)rolled surface. Starting with values $r$ which correspond to the topmost layer from $\left[\theta_{\mathrm{A}}, \theta_{\mathrm{B}}\right\rangle$, and followed by the topmost layer from $\left[\theta_{\mathrm{A}}, \theta_{\mathrm{B}}\right\rangle$, we continue until reaching the bottommost layer from $\left[\theta_{\mathrm{A}}, \theta_{\mathrm{B}}\right\rangle$. The outcome is a sequence of points $\left(\theta_{i}, r_{i}\right)$ which lie on the rolled surface. Here, $\theta_{i}$ ara (re-arranged) angles from the two intervals, and $r_{i}$ are the corresponding (and re-arranged) results of the layered surface detection.

\section{Unrolling a smooth surface of microelectronic}

The rolled surface of the microelectronic component, which we are virtually unrolling, is smooth at a large scale. However, a subtle geometric texture, originating from the weaved polymer fabric, is superimposed on the smooth surface. Furthermore, parts of the surface are coated by a thin metal layer. This has motivated a two-step approach, were we first detect an overall smooth surface.

In our proposed pipeline, the detection of the smooth surface is initiated in one slice, say a slice $z=1$, with the user manually selecting the starting and the ending point of the rolled surface, giving us angles $\theta_{\mathrm{A}}$ and $\theta_{\mathrm{B}}$. The initial slice is then unwrapped and split in two parts according to starting and ending angles. Furthermore, before using the layered surface detection, the user needs to provide the number of times the surface is rolled. 
For defining an on-surface cost function, we utilize the fact that the polymer fabric has a characteristic gray intensity, compared to darker air and brighter metal. As explained in Sec. II-B this motivates us to use the expression

$$
c_{\text {smooth }}=1-\exp \left(-\left(U-\mu_{\text {polymer }}\right)^{2}\right),
$$

where $\mu_{\text {polymer }}$ is the gray level of the polymer, and $U$ is an unwrapped slice.

Through virtual unrolling, as sketched above, we obtain a sequence of points $\left(r_{i 1}, \theta_{i 1}, z_{1}\right)$ which define the rolled surface in the initial slice. The index $i$ indicates the direction along the surface in this single slice. These points are transformed back to the Cartesian coordinate system yielding the sequence $\left(x_{i 0}, y_{i 0}, z_{0}\right)$.

While layered surface detection imposes smoothness to the results, the detected surface will still exhibit small (and discrete) leaps. We therefore additionally smooth each surface point trough weighted contributions from its neighbours along the $i$ direction. The weights are defined to minimize a combination of two terms: elasticity (length) and rigidity (curvature) [23], while making sure that end points remain fixed. For better stability, we use a backward Euler step when smoothing.

In our current implementation, the volume is processed one slice at a time. While this works well for unrolling microelectronics, for data with a higher level of noise, robustness might be improved by processing all slices at once.

When processing each subsequent slice we use the current result to initiate unwrapping. That is, instead of sampling the next slice along radial rays, we perform unwrapping by sampling along rays normal to an already detected surface in the previous slice. Since the surface moves only slightly between slices, the unwrapped image will have the surface located close to the middle of the image (vertically). Such an unwrapped image $U$ is very elongated in the direction of the surface and might be challenging to visualize. However, in this way we only need to detect one layer, and we avoid repeatedly dealing with multiple layers of the rolled surface.

As for the cost function when processing the whole volume, the expression (1) is yielding reasonable results. However, we observed that the outcome improves when the cost function is filtered with a Gaussian kernel in the direction of the surface, and with the difference of Gaussian in the direction orthogonal to the surface. As such, we are actually detecting the transition between the brighter polymer/metal surface and the darker air.

When all slices are processed, the final outcome of the surface detection is a collection of points $\left(x_{i j}, y_{i j}, z_{j}\right)$, where $j$ gives the direction of the volume slices. Indices $i$ and $j$ induce a neighbourhood relation between the detected points, leading to a quadrilateral (quad) mesh representation of the rolled surface.

For inspection of a conductive layer coated onto the rolled polymer fabric, it is important to accurately estimate distances, areas and angles. Therefore we reduce the distortion of the unrolled image by distributing the surface points along the $i$ direction at the distance of approximately one pixel. Further-

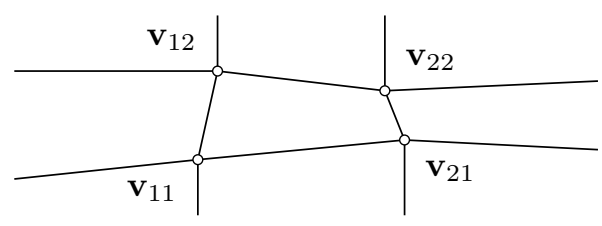

Fig. 2. A single quad element and its vertices, as used for measuring the quality (distortion) of the quad mesh. Note that in $3 \mathrm{D}$, the vertices of a quad element are generally not coplanar.

more, to eliminate skew, we apply another smoothing step, this time along the $j$ direction.

To produce an image showing the lithographically coated metal on the unrolled surface, we collect the intensities of the volumetric data in a thin volume around the smooth surface. Then we average the intensities in the direction normal to the surface. The resulting image is extremely elongated, since the surface is rolled many times.

\section{E. Quality measures for smooth surface}

We have taken heuristic steps to ensure that the quad elements of the detected mesh are close to quadratic. This was to reduce the distortion of the unrolled image, for example stretching introduced by elongated quad elements or jaggedness introduced by skewed quad elements. Here we define the measures used for assessing the quality of the quad mesh.

We assess mesh quality by calculating aspect ratio, area, and skew of every quad element. If we denote the $3 \mathrm{D}$ coordinates of the vertices defining a quad element $\mathbf{v}_{11}, \mathbf{v}_{12}, \mathbf{v}_{21}$ and $\mathbf{v}_{22}$ as in Fig. 2, the quality measures are defined by

$$
\begin{aligned}
q_{\text {aspect }} & =\frac{\left\|\mathbf{v}_{12}-\mathbf{v}_{11}+\mathbf{v}_{22}-\mathbf{v}_{21}\right\|}{\left\|\mathbf{v}_{21}-\mathbf{v}_{11}+\mathbf{v}_{22}-\mathbf{v}_{12}\right\|} \\
q_{\text {area }} & =\frac{1}{4}\left\|\mathbf{v}_{12}-\mathbf{v}_{11}+\mathbf{v}_{22}-\mathbf{v}_{21}\right\| \cdot\left\|\mathbf{v}_{21}-\mathbf{v}_{11}+\mathbf{v}_{22}-\mathbf{v}_{12}\right\| \\
q_{\text {skew }} & =\frac{\left\|\mathbf{v}_{21}-\mathbf{v}_{12}\right\|}{\left\|\mathbf{v}_{22}-\mathbf{v}_{11}\right\|} .
\end{aligned}
$$

In the expression for the aspect ratio and the area, we consider the total length of the opposite pairs of quadrilateral sides. Skew is defined as the ratio between the length of the diagonals. An ideal quad element would have an aspect ratio of 1 , area of 1 and a skew of 1 .

\section{F. Detecting subtle coarseness on microelectronic surface}

The detected smooth surface captures a large-scale geometry of the rolled surface. However, due to extensive smoothing, it will not capture the small-scale geometry. For that reason, we proceed with the second step of our pipeline, where we capture the subtle texture of the polymer fabric, as well as the thickness of the polymer substrate and the metal layer. This is potentially useful for the quantification of the rolled structure.

Here we detect three surfaces simultaneously: a boundary between the air and the polymer, a boundary between the polymer and the metal, and a boundary between the metal and the air. In areas without metal coating, the two last surfaces are coinciding. 

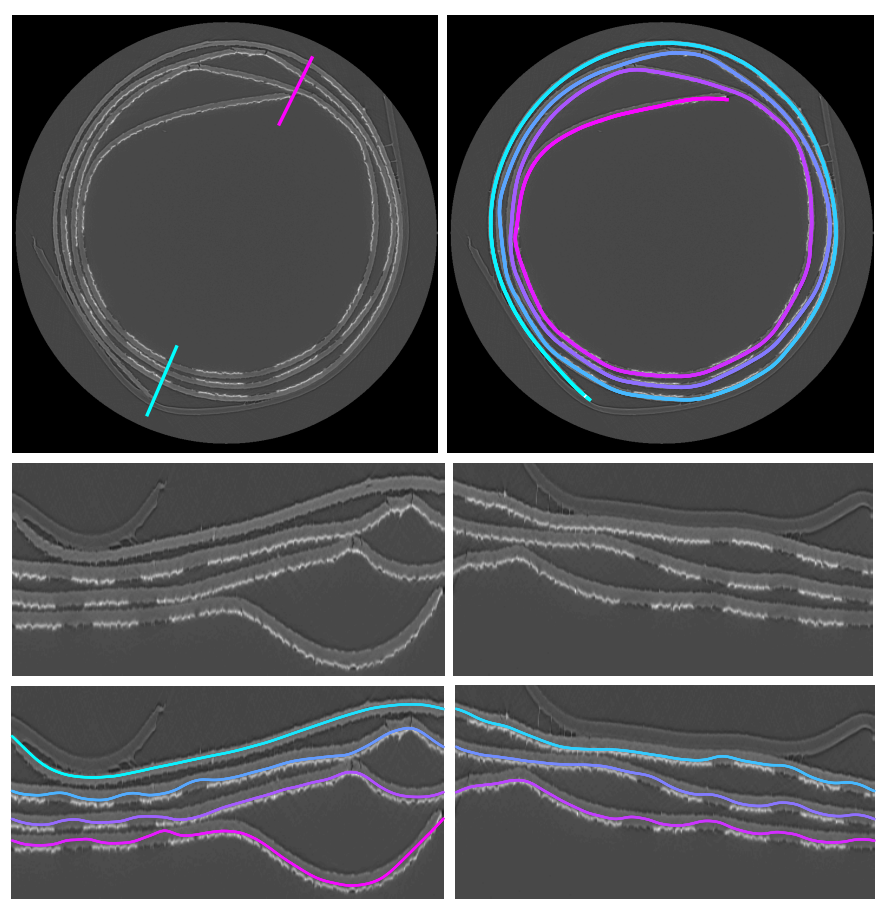

Fig. 3. Processing a first slice, shown in $x y$ domain and in $\theta r$ (unwrapped) domain. The top left image shows a slice of a volume with the starting and the ending angle as indicated by the user. The second row shows the unwrapped images, with four layers in the left image and three layers in the right. Note that the rolled microelectronic is held in place using a piece of adhesive tape, which is also visible as a layer in the image. The bottom row shows the detected layers. In the top row on the right, the detected surface is wrapped back into a $x y$ domain.

The second surface detection step is initiated around the previously found smooth surface of the microelectronics. This gives us an excellent starting position, and we constrain the detection, such that the returned surfaces only slightly deviate from the smooth surface. Furthermore, we use restrictive overlap constrains, where we utilize a relatively constant thickness of the polymer layer. For the metal layer we constrain the thickness so that it does note exceed an upper limit, but can be 0 in areas without the metal coating.

Similarity to the first step, the second step processes volumetric data slice by slice. As for the on-surface cost functions, we utilize the abrupt change in intensity between the different materials.

\section{RESULTS}

To exemplify our approach we perform a virtual unrolling of a microelectronic component consisting of a rolled polymer fabric with a thin metal layer. The object was scanned using a laboratory X-ray micro CT scanner, yielding a 3D volume of $1000^{3}$ voxels.

Fig. 3 shows processing of the first slice, which involves a limited manual input (a click on each of the two end points). Following this, all slices of the volume are processed, resulting in a smooth 3D surface. The second step of the algorithm involves detecting layers in the material, as shown in Fig. 4. Note the successful separation of the densely packed layers.

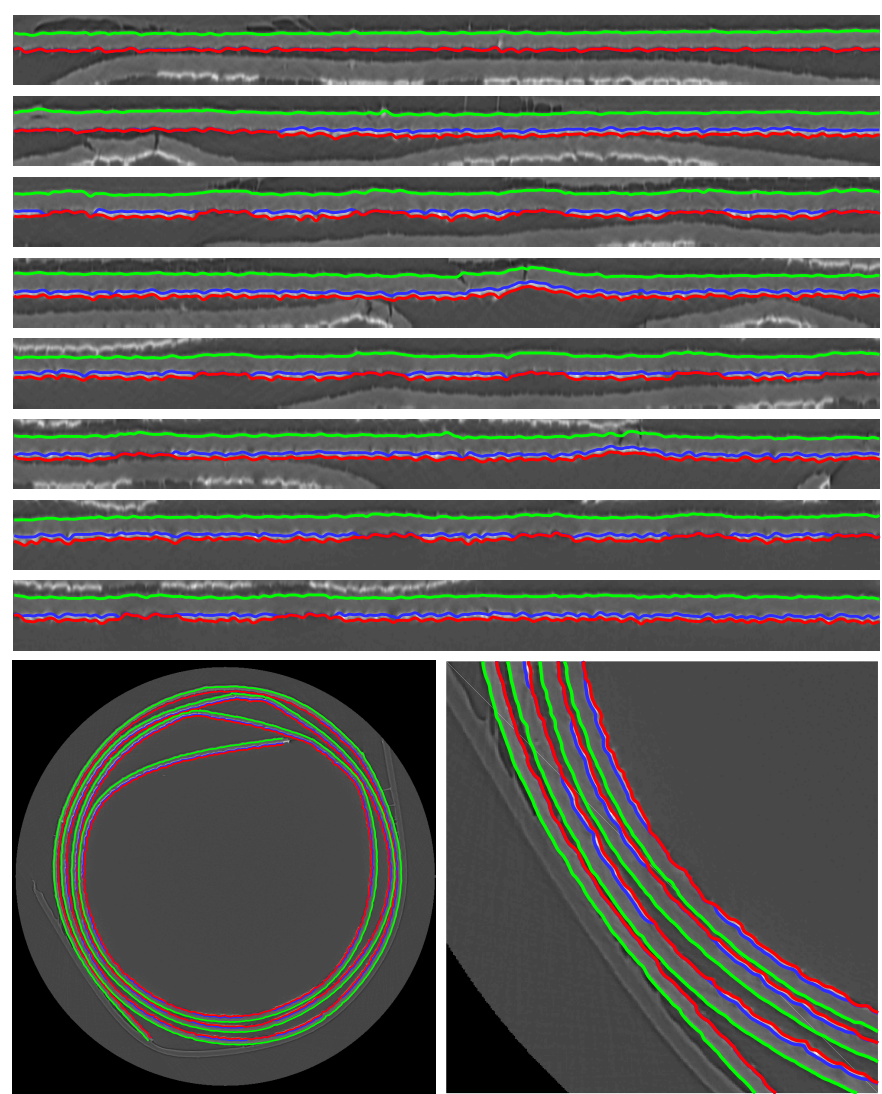

Fig. 4. Simultaneously detecting three surfaces, shown in $x y$ domain and in an unwrapped image (the elongated image is shown broken in 8 pieces). The first surface (red) is a surface of the metal layer where metal coating is present, or of the polymer in areas without coating. The next (blue) surface is an interface between the metal and the polymer layer; in the area without coating this surface coincides with the first surface. The third surface (green) is the bottom layer of the polymer sheet.

Furthermore, all surface are connected despite the cracks in the polymer.

The intensity image of the unrolled surface is shown in Fig. 5. The areas of the surface which are coated by the metal are clearly visible. We can also notice the weaved texture of the polymer fabric. Thanks to the high quality of the obtained quad mesh, there is practically no distortion in the image which is caused by virtual unrolling, and the surface structures (for example cracks in the conductive layer) can be quantified with high accuracy. The conductive layer of the piece we scanned exhibited no critical cracks, but we observed some cracking both in the polymer layer and in the metal coating.

Fig. 6 shows the detected surfaces in 3D. First we show the smooth surface of the rolled microelectronic as a quad mesh, where we for the sake of visualization significantly reduce mesh resolution: the original mesh has 144 times more quad faces. The next two 3D visualizations show the surface found in the second step of the algorithm. To appreciate the subtle geometric detail of the surface found in the second step of the algorithm, we first visualize the textured surface without the intensity information. Notice how the geometric texture reveals the weaved structure of the polymer fabric. Lastly, we 
also show this surface with the intensity information on top of the fine texture.

Lastly, in Fig. 7 we show distribution histograms for the three mesh quality measures: aspect ratio, area and skew. Those are collected by considering all quad elements of the mesh. We also show the mean and the standard deviation for each quality measure. The distributions are close to the ideal quad quality (aspect ratio of 1 , area of 1 and skew of 1) and rather narrow. Therefore, the quality measures indicate a minimal distortion of the unrolled image. Furthermore, these measures can be used for assessing the accuracy of the quantifications performed on the unrolled images.

\section{CONCLUSION}

We suggest an automated method for virtual unrolling of a imaged rolled object. Our method utilizes a very constrained geometry of the problem, which strongly reduces the solutions space of the surface detection problem. As such, our method is extremely robust and can successfully resolve touching layers of the rolled surface.

Our approach is motivated by the need of inspecting a rolled piece of microelectronics, and in this paper we demonstrate how our method can be used for this purpose.

The surface we detect is represented as a quad mesh of a high resolution and an extremely high quality. This allows for a very accurate quantification of the structures on the unrolled surface and makes our approach widely applicable, also for industrial use.

\section{REFERENCES}

[1] C. J. Kennedy and T. J. Wess, "The use of X-ray scattering to analyse parchment structure and degradation," Physical Techniques in the Study of Art, Archaeology and Cultural Heritage, vol. 1, pp. 151-172, 2006.

[2] C. Liu, P. L. Rosin, Y.-K. Lai, and W. Hu, "Robust segmentation of historical parchment xmt images for virtual unrolling," in Digital Heritage, vol. 1. IEEE, 2015, pp. 11-18.

[3] V. Mocella, E. Brun, C. Ferrero, and D. Delattre, "Revealing letters in rolled herculaneum papyri by $\mathrm{x}$-ray phase-contrast imaging," Nature communications, vol. 6, p. 5895, 2015.

[4] I. Bukreeva, A. Mittone, A. Bravin, G. Festa, M. Alessandrelli, P. Coan, V. Formoso, R. Agostino, M. Giocondo, F. Ciuchi et al., "Virtual unrolling and deciphering of herculaneum papyri by $\mathrm{x}$-ray phase-contrast tomography," Scientific reports, vol. 6, p. 27227, 2016.

[5] D. Allegra, E. Ciliberto, P. Ciliberto, G. Petrillo, F. Stanco, and C. Trombatore, "X-ray computed tomography for virtually unrolling damaged papyri," Applied Physics A, vol. 122, no. 3, p. 256, 2016.

[6] D. Baum, N. Lindow, H.-C. Hege, V. Lepper, T. Siopi, F. Kutz K. Mahlow, and H.-E. Mahnke, "Revealing hidden text in rolled and folded papyri," Applied Physics A, vol. 123, no. 3, p. 171, 2017.

[7] O. Samko, Y.-K. Lai, D. Marshall, and P. L. Rosin, "Virtual unrolling and information recovery from scanned scrolled historical documents," Pattern Recognition, vol. 47, no. 1, pp. 248-259, 2014.

[8] W. B. Seales, J. Griffioen, R. Baumann, and M. Field, "Analysis of herculaneum papyri with x-ray computed tomography," in International Conference on Nondestructive Investigations and Microanalysis for the Diagnostics and Conservation of Cultural and Environmental Heritage, 2011.

[9] D. Allegra, E. Ciliberto, P. Ciliberto, F. L. M. Milotta, G. Petrillo, F. Stanco, and C. Trombato, "Virtual unrolling using x-ray computed tomography," in 23rd European Signal Processing Conference. IEEE, 2015, pp. 2864-2868.

[10] O. Samko, Y.-K. Lai, A. D. Marshall, and P. L. Rosin, "Segmentation of parchment scrolls for virtual unrolling." in British Machine Vision Conference, 2011, pp. 1-11.

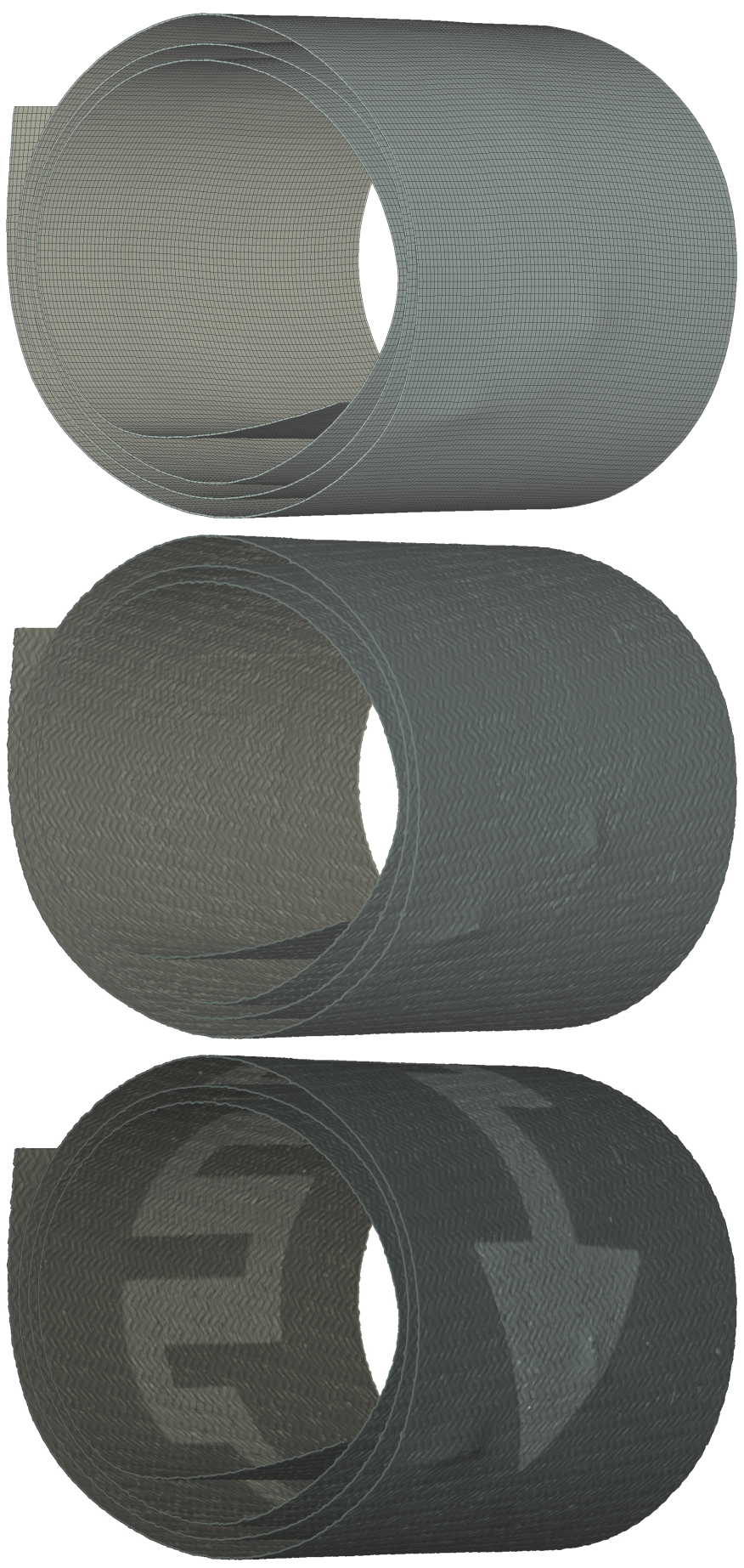

Fig. 6. Detected surfaces in 3D. In the top row a smooth surface is shown as a quad mesh. For better visualization, we have reduced the mesh resolution by merging an area covered by $12 \times 12$ quad elements into a single quad face. We have also visualized a single surface as a very thin layer. In the middle row we show a rendering of the mesh which captures the subtle geometric texture of the rolled surface. This mesh contains $997 \times 8120$ vertices and contains only geometry (not color) information. In the bottom row we added the intensity (color) information from the image in Fig. 5 to the textured mesh. 

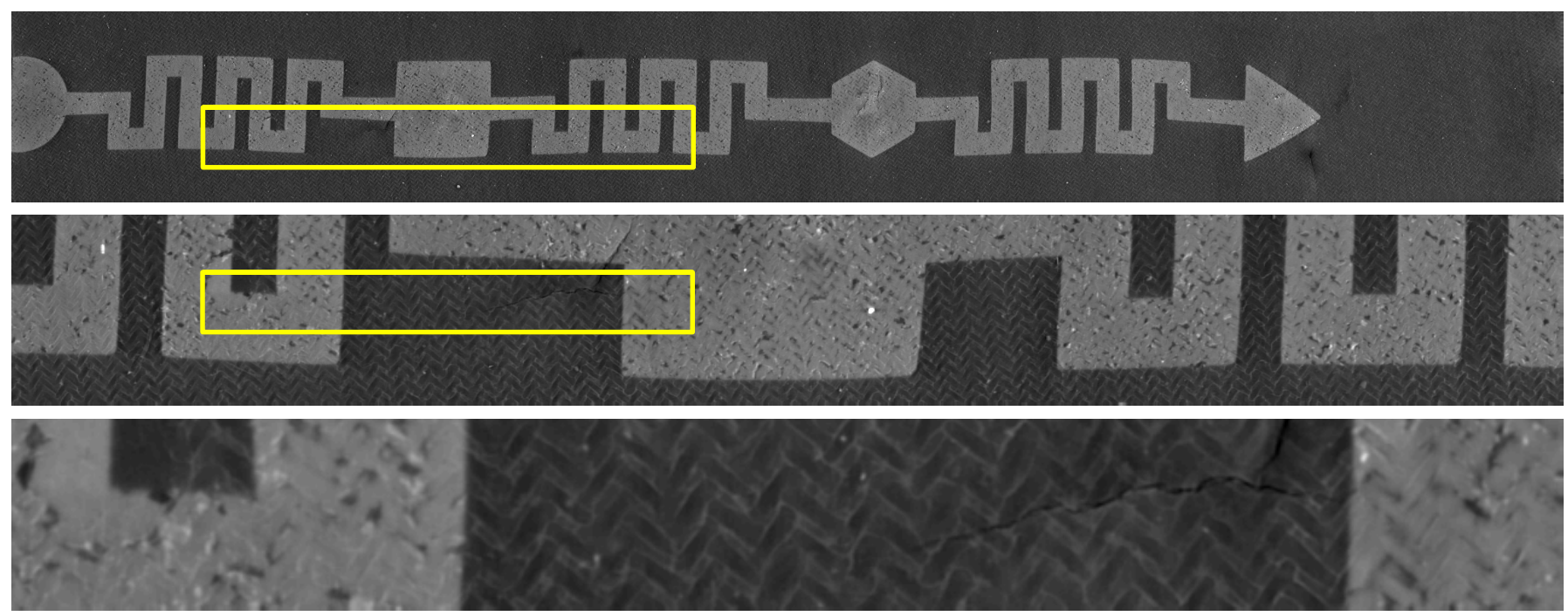

Fig. 5. Unrolled image of the microelectronic sheet. The resulting image contains $997 \times 8120$ pixels, and to appreciate a high resolution of the result, we show two degrees of zoom-in: the first covering a region of $315 \times 2562$ pixels, and the second covering $100 \times 812$ pixels. Note a visible crack in the material.
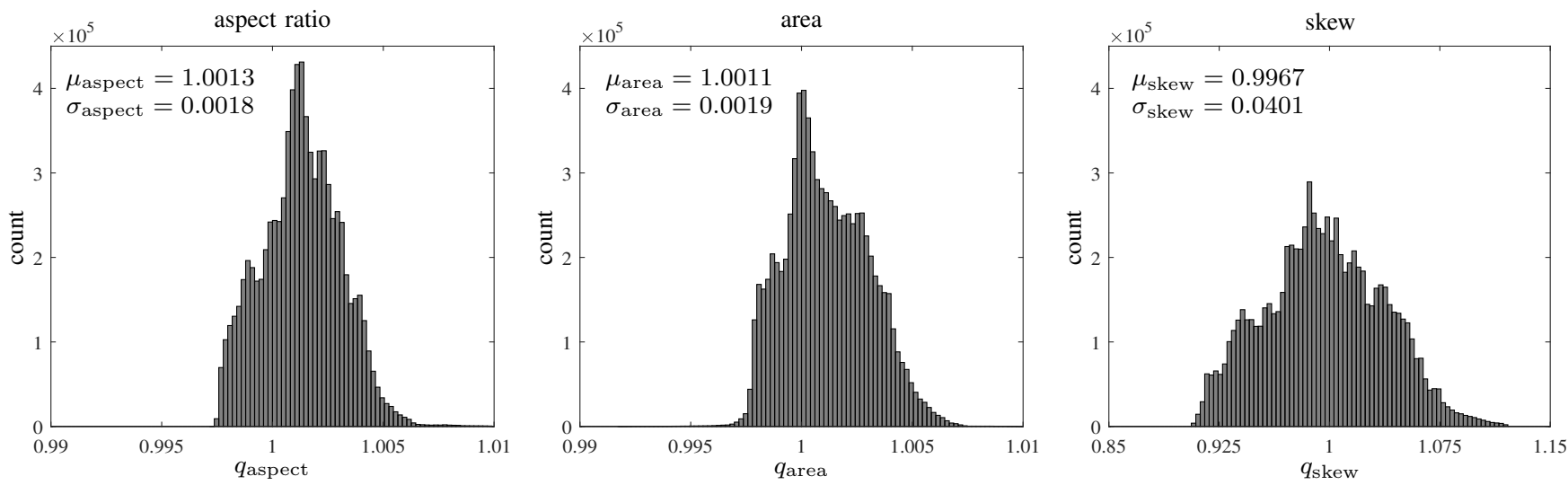

Fig. 7. Quality measures for quadrilateral mesh. The histograms are produced by considering every quad element in the mesh. For each quality measure we also show the mean $(\mu)$ and the standard deviation $(\sigma)$ over all elements. From left to right: aspect ratio, area and skew of the quad elements.

[11] X. Wu and D. Z. Chen, "Optimal net surface problems with applications," in Automata, Languages and Programming. Springer, 2002, pp. $1029-1042$.

[12] K. Li, X. Wu, D. Z. Chen, and M. Sonka, "Optimal surface segmentation in volumetric images - a graph-theoretic approach," IEEE Transactions on Pattern Analysis and Machine Intelligence, vol. 28, no. 1, pp. 119134, 2006.

[13] M. K. Garvin, M. D. Abràmoff, R. Kardon, S. R. Russell, X. Wu, and M. Sonka, "Intraretinal layer segmentation of macular optical coherence tomography images using optimal 3-d graph search," IEEE Transactions on Medical Imaging, vol. 27, no. 10, pp. 1495-1505, 2008.

[14] J. Petersen, P. Lo, M. Nielsen, G. Edula, H. Ashraf, A. Dirksen, and M. de Bruijne, "Quantitative analysis of airway abnormalities in ct," in SPIE Medical Imaging. International Society for Optics and Photonics, 2010, pp. 76241S-76241S.

[15] M. Haeker, X. Wu, M. Abràmoff, R. Kardon, and M. Sonka, "Incorporation of regional information in optimal 3-d graph search with application for intraretinal layer segmentation of optical coherence tomography images," in Information Processing in Medical Imaging. Springer, 2007, pp. 607-618.

[16] X. Wu, D. Z. Chen, K. Li, and M. Sonka, "The layered net surface problems in discrete geometry and medical image segmentation," International Journal of Computational Geometry \& Applications, vol. 17, no. 03, pp. 261-296, 2007.
[17] M. Haeker, M. D. Abràmoff, X. Wu, R. Kardon, and M. Sonka, "Use of varying constraints in optimal 3-d graph search for segmentation of macular optical coherence tomography images," in International Conference on Medical Image Computing and Computer-Assisted Intervention. Springer, 2007, pp. 244-251.

[18] Q. Song, J. Bai, M. K. Garvin, M. Sonka, J. M. Buatti, and X. Wu, "Optimal multiple surface segmentation with shape and context priors," IEEE Transactions on Medical Imaging, vol. 32, no. 2, pp. 376-386, 2013.

[19] V. A. Dahl, A. B. Dahl, and R. Larsen, "Surface detection using round cut," in 2nd International Conference on 3D Vision, vol. 2. IEEE, 2014, pp. $82-89$.

[20] O. Lézoray and L. Grady, Image processing and analysis with graphs: theory and practice. CRC Press, 2012.

[21] L. R. Ford and D. R. Fulkerson, "Maximal flow through a network," Canadian Journal of Mathematics, vol. 8, no. 3, pp. 399-404, 1956

[22] Y. Boykov and V. Kolmogorov, "An experimental comparison of mincut/max-flow algorithms for energy minimization in vision," IEEE Transactions on Pattern Analysis and Machine Intelligence, vol. 26, no. 9, pp. 1124-1137, 2004.

[23] C. Xu, D. L. Pham, and J. L. Prince, "Image segmentation using deformable models," Handbook of Medical Imaging, vol. 2, pp. 129174, 2000. 